\title{
ESTIMATING THE PROBABILITY OF RUIN \\ FOR VARIABLE PREMIUMS BY SIMULATION
}

\author{
By FrédéRIC Michaud \\ University of Lausanne, Switzerland
}

\begin{abstract}
There is a duality between the surplus process of classical risk theory and the single-server queue. It follows that the probability of ruin can be retrieved from a single sample path of the waiting time process of the single-server queue. In this paper, premiums are allowed to vary. It has been shown that the stationary distribution of a corresponding storage process is equal to the survival probability (with variable premiums). Thus by simulation of the corresponding storage process, the probability of ruin can be obtained. The special cases where the surplus earns interest and the premiums are charged by layers are considered and illustrated numerically.
\end{abstract}

\section{KEYWORDS}

Risk theory; Probability of ruin; Variable premiums; Interest; Premiums by layers; Simulation.

\section{INTRODUCTION}

The study of the probability of ruin has been the centre of interest of many papers treating actuarial risk theory. Most of these articles deal with the problem of determining this probability in the case of constant premiums. The mathematics are simpler and explicit results have been obtained for certain claim amount distributions. Fewer papers consider the case of premiums whose value depend on the current surplus. Some of them are Gerber (1975), TAYLOR (1980) and SCHMIDLI (1994). As one would expect, fewer explicit results have been obtained. For exponential jumps, Gerber (1975) obtains an exact expression for the ruin probability.

When no explicit solution is available, the actuary must rely on alternative tools to obtain an answer. DuFrESNE and GERBER (1989) make use of the duality between the virtual waiting time of the single-server queue $(M / G / 1)$ of queuing theory and the risk process of risk theory in the case of constant premium rates to obtain the probability of ruin by simulation. See FELLER (1971, p. 198) and SEAL (1972) for proofs of this duality. 
If the premiums are no longer assumed constant, a duality still exists between the two processes. SIEGMUND (1976) first established the conditions on the probability measure of the storage process under which a dual risk process exists. HARRISON and Resnick (1978), and Asmussen and PETERSEN (1989) have also shown the duality. The goal of this paper is to illustrate that the method of DuFrESNE and GERBER (1989) can be adapted to the case of variable premiums. Thus a single sample path of the corresponding storage process is sufficient to provide an estimate of the probability of ruin.

Two special cases will be studied. The first one considers the situation where interest is taken into account, and the other one looks at premiums charged by layers. We look at the speed of convergence of the estimate when different assumptions are made. The method proposed in this paper is put in perspective with alternative ones in the last section.

\section{THE SURPLUS PROCESS AND THE STORAGE PROCESS}

In the following, the surplus process $\{U(t) ; t \geq 0\}$ is defined by the initial value $U(0)=u$ and the stochastic differential equation

$$
d U(t)=c(U(t)) d t-d S(t),
$$

where $\{S(t) ; t \geq 0\}$ is the aggregate claims process and $c(\cdot)$ is the premium rate function such that

$$
c(u)>0 \text { for } u>0 .
$$

It is assumed that $\{S(t) ; t \geq 0\}$ is a compound Poisson process with parameter $\lambda$ and claim amount distribution $P(\cdot)$. We wish to stress the fact that the claims are not necessarily positive.

It is worth mentioning that $(2.1)$ is a quite general definition of the surplus process. The classical case is retrieved if $c(\cdot)$ is constant. If it is assumed that the surplus earns interest at a constant force $\delta$, we have

$$
c(x)=c+\delta x .
$$

Thus earning interest is in fact "equivalent" to receiving premiums at a rate which is a linear function of the surplus. Another example is the case where premiums are charged by layers. These two examples are treated in section 5 .

In order to build a dual process, let $\{X(t)\}$ be defined by an initial value $X(0)$ and the stochastic differential equation

$$
d X(t)=-c(X(t)) d t+d S(t)
$$

where $c(\cdot)$ and $S(t)$ are the same as in (2.1). In addition to (2.4), we impose on $\{X(t)\}$ the condition that it be a process with non-negative values. Thus the increments of $\{X(t)\}$ are defined by (2.4) unless they would cause the process to fall below 0 , in which case the process is equal to 0 until the next positive jump. Figure 1 illustrates a sample path of $\{X(t)\}$ and $\{U(t)\}$ with same initial value. 


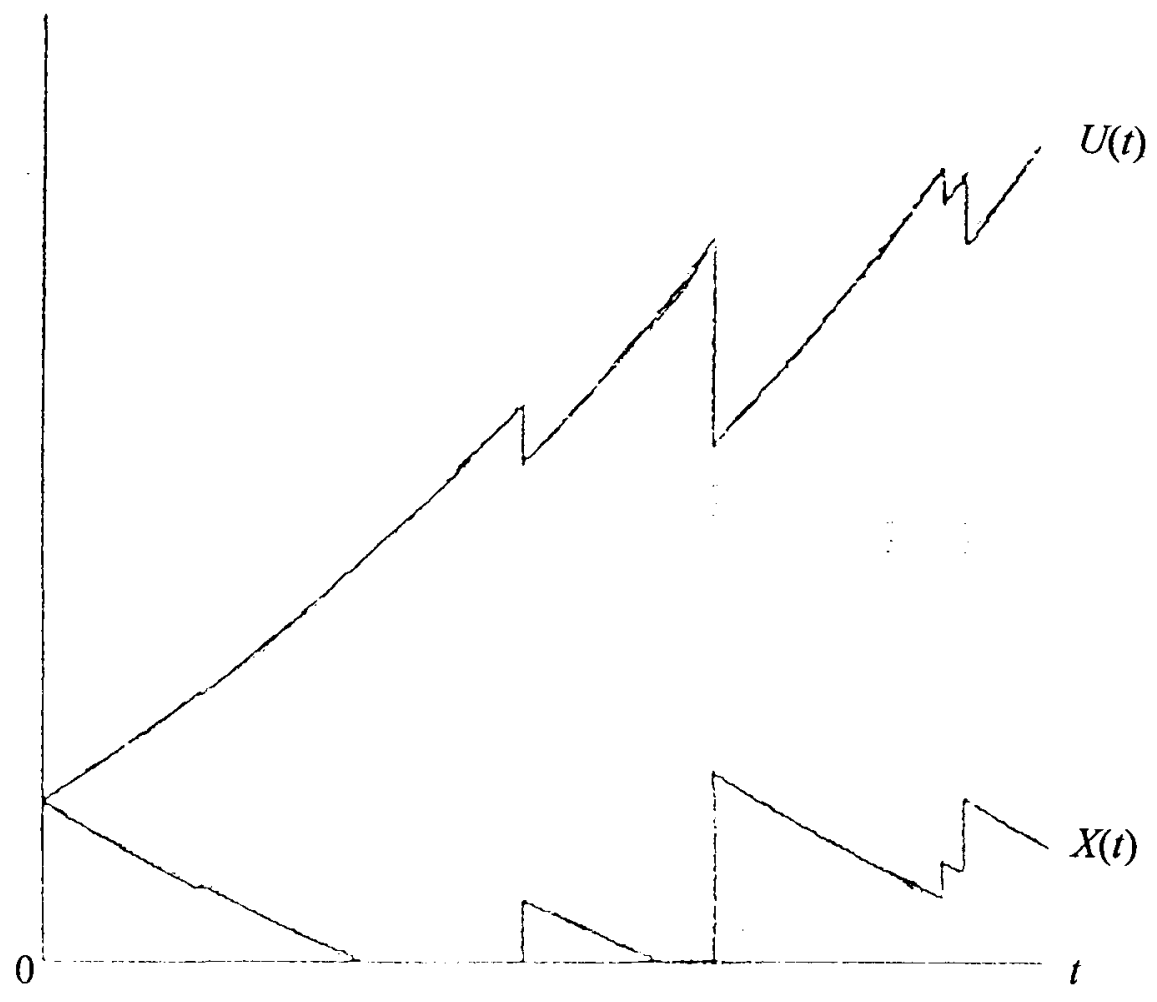

FIGURE 1. Typical sample paths of $\{X(t)\}$ and $\{U(t)\}$.

The process $\{X(t)\}$ is called a storage process due the following interpretation. Suppose that $\{X(t)\}$ is in state $x$ at time $t$ (call $x$ the stock) and consider the infinitesimal time interval $(t, t+d t)$. In that interval, there is an input of amount $d S(t)$ and an output equal to $c(x) d t$. In this context, $c(x)$ is called the release rate, which in our case is a function of the current stock. It is assumed that stocks cannot be negative. This heuristically explains (2.4).

\section{A PROOF OF THE DUALITY}

For completeness, we give a proof of the duality between the risk process and the storage process defined by $(2.1)$ and (2.4). The reader is referred to SIEGMUND (1976), Harrison and Resnick (1978) and Asmussen and PETERSEN (1989) for alternative arguments. We use the classical notation $\psi(u)$ for the probability of ruin given the initial surplus $u$, i.e.

$$
\psi(u)=\operatorname{Pr}(U(t)<0 \text { for some } t \geq 0),
$$


and define the survival probability as $\bar{\psi}(u)=1-\psi(u)$. It is shown next that $\bar{\psi}(u)$ is a solution of the integro-differential equation

$$
c(u) \bar{\psi}^{\prime}(u)=\lambda \bar{\psi}(u)-\lambda \int_{-\infty}^{u} \bar{\psi}(u-y) d P(y), u>0 .
$$

First, we define implicitly the function $u(t)$ by

$$
u(t)=u+\int_{0}^{t} c(u(s)) d s .
$$

Before the first claim, $u(t)$ and $U(t)$ will be equal. Second, consider an interval of arbitrary length $h$. Now condition on whether there is a claim between times 0 and $h$ and on the time of this possible first claim. There is no claim with probability $e^{-\lambda h}$ and a claim at time $t<h$ with probability $\lambda e^{-\lambda t} d t$. With no claim in the interval, the surplus grows to $u(h)$ at time $h$. With a claim a time $t$, the surplus becomes $u(t)-y$ at time $t$ with probability $d P(y)$. Applying the law of total probability yields

$$
\bar{\psi}(u)=e^{-\lambda h} \bar{\psi}(u(h))+\lambda \int_{0}^{h} e^{-\lambda t} \int_{-x}^{u(t)} \bar{\psi}(u(t)-y) d P(y) d t .
$$

Note that $\bar{\psi}(u)=0$ for negative values of $u$, i.e., ruin is certain when the initial surplus is negative. Taking derivatives of both sides of (3.4) with respect to $h$ and letting $h$ tend to 0 leads to equation (3.2).

Let $F(x, t)$ be the probability distribution function of the random variable $X(t)$, that is

$$
F(x, t)=\operatorname{Pr}(X(t) \leq x)
$$

From here on, it is assumed that $c(\cdot)$ is "sufficiently large" so that the process $\{X(t)\}$ does not escape to infinity and has a stationary distribution. Let $F(x)$ be the stationary distribution. Then we have (see Ross (1983), theorem 4.3.3)

$$
F(x)=\lim _{t \rightarrow x} F(x, t)
$$

With arguments similar to the ones leading to (3.2), we can derive the following integro-differential equation for $F(x)$ :

$$
c(x) F^{\prime}(x)=\lambda F(x)-\lambda \int_{-\infty}^{x} F(x-y) d P(y), x>0 .
$$

Consider the function $\xi(t)$ defined by

$$
\xi(t)=x+\int_{t}^{0} c(\xi(s)) d s
$$


and the time interval $t_{0}-h \leq t \leq t_{0}\left(0<h<t_{0}\right)$. In other words, we look backward from $t_{0}$ at an interval of length $h$. Then the event $\left(X\left(t_{0}\right) \leq x\right)$ is equivalent to one of the following events :

- $\left(X\left(t_{0}-h\right) \leq \xi(-h)\right)$ if no jumps occur in the given time interval, or

- $\left(X\left(t_{0}-\tau\right) \leq \xi(-\tau)-y\right)$ if, in the given time interval, the last jump of the sample path is of amount $y$ and occurs at time $t_{0}-\tau, \tau<h$.

Thus, by the law of total probability, we have

$$
\begin{aligned}
F\left(x, t_{0}\right)= & e^{-\lambda h} F\left(\xi(-h), t_{0}-h\right) \\
& +\lambda \int_{0}^{h} e^{-\lambda \tau} \int_{-\infty}^{\xi(-\tau)} F\left(\xi(-\tau)-y, t_{0}-\tau\right) d P(y) d \tau .
\end{aligned}
$$

Taking limits as $t_{0}$ tends to infinity and successively applying the Dominated Convergence Theorem (RUDIN (1976), p. 321; note that $\left|e^{-\lambda \tau}\right|$ and $|F(\cdot)|$ are bounded by 1) yields

$$
F(x)=e^{-\lambda h} F(\xi(-h))+\lambda \int_{0}^{h} e^{-\lambda \tau} \int_{-\infty}^{\xi(-\tau)} F\left(\xi(-\tau)-y, t_{0}-\tau\right) d P(y) d \tau .
$$

Taking derivatives with respect to $h$ and letting $h$ tend to 0 gives us (3.7).

If we compare (3.2) and (3.7), we see that $\bar{\psi}(x)$ and $F(x)$ are solutions of the same integro-differential equation. In conjunction with the conditions $F(\infty)=1$ and $\bar{\psi}(\infty)=1$, these equations have a unique solution (see DUFresne and Gerber (1989), p. 78), and we conclude that $F(x)=\bar{\psi}(x)$. Thus we can estimate the probability of ruin by estimating $F(x)$.

\section{Remark}

In the proof of (3.2), it must be kept in mind that $\bar{\psi}(u)$ is not differentiable at the points of discontinuity of $c(u)$. However, note that the expression on the right-hand side of (3.2) is a continuous function of $u$ and it follows that the left-hand side is also continuous. Thus, although $\bar{\psi}^{\prime}(u)$ does not exist at points of discontinuity of $c(u)$, the product $\bar{\psi}^{\prime}(u) c(u)$ is continuous. This remark holds true for (3.7) and $F(x)$.

\section{STATIONARY DISTRIBUTION AND RUIN PROBABILITY}

Following the reasoning of DUfresne and GERBER (1989), define $D(x, t)$ as the total time that the process $\{X(t)\}$ spends below level $x$ before time $t$. From (3.6) we have

$$
\lim _{t \rightarrow \infty} \frac{D(x, t)}{t}=F(x) \text { almost surely. }
$$

Heuristically, (4.1) means that in the long run, the probability that a chosen point of the sample path is below $x$ is equal to the proportion of time spent by the process 
below $x$. This intuitively makes sense. A proof of (4.1) can be found in HOEL, PORT and STONE (1972) (Theorem 1, p. 58), which sums up to an application of the strong law of large numbers. Hence, $F(x)$ and the probability of ruin can be obtained with the simulation of a single sample path of $\{X(t)\}$.

Still along the idea of DUFRESNE and Gerber (1989), one way to keep track of the total time spent below level $x$ by a sample path is to consider the random variables $D_{n}(x)$, which are defined as the total time spent below $x$ before the $n$th jump. If $T_{n}$ is the random time elapsed between the $(n-1)$ th and $n$th jump, we have that

$$
\lim _{n \rightarrow \infty} \frac{D_{n}(x)}{\sum_{i=1}^{n} T_{i}}=F(x) \text { almost surely }
$$

since $\lim _{n \rightarrow \infty} \sum_{i=1}^{n} T_{i}=\infty$ almost surely.

\section{ILLUSTRATION}

According to (4.2), if we perform a sufficiently large number of simulations, the convergence of the estimate to $F(x)$ is certain. Still we must test how fast this convergence occurs. We will look at two cases for the premium function $c(\cdot)$. The first consists in allowing the surplus to earn interest and the second one treats the case of premiums by layers.

\subsection{Interest on the surplus}

Recall (2.3) which gave as a premium function

$$
c(u)=c+\delta u .
$$

We will simulate sample paths of the process $\{X(t)\}$ defined by the arbitrarily chosen initial value $X(0)=0$ and

$$
d X(t)=-(c+\delta X(t)) d t+d S(t) .
$$

If we look at the process between jumps, then solving $X^{\prime}(t)=-\mathrm{c}-\delta X(t)$ yields

$$
X(t)=X\left(t_{0}\right) e^{-\delta\left(t-t_{0}\right)}-c\left(\frac{1-e^{-\delta\left(t-t_{0}\right)}}{\delta}\right), t>t_{0} .
$$

First, we simulate jump amounts $Y_{1}, Y_{2}, \ldots$, and interjump times $T_{1}, T_{2}, \ldots$, where $Y_{i}$ is the amount of the $i$ th jump and $T_{i}$ is the time between jumps $Y_{i-1}$ and $Y_{i} . T_{1}$ is defined as the time before the first jump. Then, to keep track of the process, let $X_{i}$ 
be the value of the process immediately after jump $i$, with $X_{0}=0$. Keeping in mind that negative jumps are allowed and that $\{X(t)\}$ is non-negative, we get with (5.3) that

$$
X_{n}=\max \left[0, Y_{n}, X_{n-1} e^{-\delta T_{n}}-c\left(\frac{1-e^{-\delta T_{n}}}{\delta}\right)+Y_{n}\right]
$$

The amount of time $D_{n}(x)$ spent by the process below level $x$ before the $n$th jump is obtained recursively by

$$
D_{n+1}(x)= \begin{cases}D_{n}(x)+T_{n+1}, & X_{n} \leq x, \\ D_{n}(x)-\left(T_{n+1}-r_{n+1}\right)_{+}, & X_{n}>x,\end{cases}
$$

with $D_{0}(x)=0, x_{+}=\max (0, x)$, and $r_{n+1}$ being the time needed to reach level $x$ starting from $X_{n}$ (assuming no jumps occur during this time interval). The period $r_{n+1}$ is implicitly defined by $t-t_{0}$ in (5.3) with $X\left(t_{0}\right)=X_{n}$ and $X(t)=x$. Thus

$$
r_{n+1}=\frac{1}{\delta} \ln \left(\frac{X_{n}+c / \delta}{x+c / \delta}\right)
$$

Table 1 gives estimates of the probability of ruin for different numbers of simulations $n$ and different initial surplus values $u$. The Poisson parameter is $\lambda=1$ and the jump amount distribution is exponential with mean equal to 1 . The premium $c$ is equal to 1 and the force of interest $\delta$ is 0.05 . Thus no security margin is included in the premium. Although in the classical model ruin is certain when no security margin is included in the premium, it is not the case when the surplus yields interest. Moreover, it can be seen that if the initial surplus is sufficiently high, the probability of ruin becomes quite small, even in the absence of a security margin. The exact values at the bottom of each column are given by the formula

$$
\psi(u)=\frac{\Gamma\left(\frac{\lambda}{\delta}, \frac{c}{\delta \mu}+\frac{u}{\mu}\right)}{\Gamma\left(\frac{\lambda}{\delta}, \frac{c}{\delta \mu}\right)+\frac{\delta}{\lambda}\left(\frac{c}{\delta \mu}\right) \frac{\lambda}{\delta} e^{-\frac{c}{\delta \mu}}},
$$

with the incomplete gamma function defined as

$$
\Gamma(a, b)=\int_{b}^{\infty} x^{a-1} e^{-x} d x
$$

Formula (5.7) is attributed to Segerdahl (1942). 
TABLE 1

THE PROBABILITY OF RUIN OBTAINED BY SIMULATION WITH $\lambda=1, c=1, \delta=0.05, P(x)=1-e^{-x}, x>0$.

\begin{tabular}{l|llllll}
\hline \hline$n \backslash u$ & 0 & 2 & 4 & 6 & 8 & 10 \\
\hline 10000 & 0.848728 & 0.552233 & 0.334032 & 0.190215 & 0.100786 & 0.052822 \\
100000 & 0.846427 & 0.553203 & 0.326112 & 0.177550 & 0.087981 & 0.041057 \\
200000 & 0.844146 & 0.550787 & 0.325396 & 0.176152 & 0.086685 & 0.039624 \\
300000 & 0.843202 & 0.549879 & 0.324983 & 0.175151 & 0.086354 & 0.039693 \\
400000 & 0.842386 & 0.548004 & 0.321891 & 0.172704 & 0.085010 & 0.039081 \\
500000 & 0.842270 & 0.547173 & 0.321128 & 0.171949 & 0.084398 & 0.038676 \\
600000 & 0.841583 & 0.545989 & 0.319980 & 0.171063 & 0.083841 & 0.038345 \\
700000 & 0.841850 & 0.546447 & 0.320371 & 0.171678 & 0.084360 & 0.038706 \\
800000 & 0.841910 & 0.545976 & 0.319828 & 0.171489 & 0.084383 & 0.038660 \\
900000 & 0.841728 & 0.546393 & 0.320642 & 0.171790 & 0.084402 & 0.038724 \\
1000000 & 0.841819 & 0.546590 & 0.320795 & 0.171867 & 0.084570 & 0.038740 \\
exact & 0.841108 & 0.547364 & 0.322416 & 0.173175 & 0.085508 & 0.039123 \\
\hline
\end{tabular}

Table 1 seems to indicate that in this case, simulating 1000000 claims provides an estimate with a precision of about \pm 0.001 . One way to verify any statement about convergence is to carry out the simulation a given number of times and then compute the sample variance of the estimates for each $n$ and $u$. However, statistical common sense suggests that it is not necessary to verify the sample variance for each $n$. The reason for this lies in the fact that our estimates are, more or less, just sample means. Thus using one sample of size $m n$ or $m$ samples of size $n$ should give similar values for the estimated variance of the population. In other words, estimating $F(x)$ with 1000000 claims should provide a value as precise as taking the sample mean of 100 estimates of $F(x)$ with 10000 claims. (Since $m$ samples have equal initial values, a fact which does not hold if we "split" into $m$ one larger sample, these statements are not exactly true in our case. However, a few tests should convince the reader that the bias is small and of no practical importance.) Table 2 gives sample means and standard deviations for estimates of $F(x)$ obtained from 100 simulations of 10000 claims as done in Table 1.

TABLE 2

SAMPLE MEANS AND STANDARD DEVIATIONS OF THE FIRST ROW OF TABLE 1.

\begin{tabular}{l|llllll}
\hline \hline$u$ & 0 & 2 & 4 & 6 & 8 & 10 \\
\hline mean & 0.840817 & 0.546415 & 0.321480 & 0.173301 & 0.086320 & 0.039825 \\
std. dev. & 0.006811 & 0.015135 & 0.016091 & 0.012874 & 0.009071 & 0.006142 \\
\hline
\end{tabular}

Given the estimated values of the standard deviation in the last row of Table 2, we know approximately how many claims we must simulate in order to get a desired precision. A $95.44 \%$ confidence interval for the exact value of $F(x)$ is given by

$$
\left[F_{n}(x)-2 s_{n}(x), F_{n}(x)+2 s_{n}(x)\right]
$$


where $F_{n}(x)$ is the estimate of $F(x)$ and $s_{n}(x)$ is the sample standard deviation when $n$ claims are simulated. The values of $s_{n}(x)$ can be approximated by the values of Table 2 divided by $\sqrt{n / 10000}$. In the next section, we shall return to the question of efficiency and usefulness of this method.

One should note that the standard deviation for $u=4$ is almost twice the one for $u=10$. Thus the value of $u$ has an effect on the precision of the estimate. This makes sense since for high enough values of $u$, the estimate for $F(x)$ tends to zero. Hence we must expect that the standard deviation should at some point start decreasing and reach zero for high values of $u$.

Table 3 and Table 4 give results for a case similar to Table 1 and Table 2, with the exception that the premium $c$ is now set equal to 1.5 . The reader is invited to compare the estimates when $n=10000$ with the exact value. The estimates are already about exact to the second decimal place. It is a consequence of the smaller standard deviations.

TABLE 3

THE PROBABILITY OF RUIN OBTAINED BY SIMULATION WITH $\lambda=1, c=1.5, \delta=0.05, P(x)=1-e^{-x}, x>0$.

\begin{tabular}{c|llllll}
\hline \hline$n \backslash u$ & 0 & 2 & 4 & 6 & 8 & 10 \\
\hline 10000 & 0.626483 & 0.267620 & 0.103953 & 0.039879 & 0.013643 & 0.003166 \\
100000 & 0.621247 & 0.267299 & 0.108216 & 0.041382 & 0.015142 & 0.005366 \\
200000 & 0.621558 & 0.266750 & 0.108240 & 0.041695 & 0.015280 & 0.005514 \\
300000 & 0.620434 & 0.265071 & 0.106970 & 0.041265 & 0.015145 & 0.005478 \\
400000 & 0.620350 & 0.265389 & 0.107332 & 0.041506 & 0.015226 & 0.005473 \\
500000 & 0.621022 & 0.266831 & 0.108398 & 0.041920 & 0.015207 & 0.005330 \\
600000 & 0.620897 & 0.266538 & 0.108153 & 0.041820 & 0.015248 & 0.005372 \\
700000 & 0.620798 & 0.266677 & 0.107939 & 0.041564 & 0.015010 & 0.005222 \\
800000 & 0.620505 & 0.266258 & 0.107762 & 0.041427 & 0.014983 & 0.005195 \\
900000 & 0.620099 & 0.265688 & 0.107292 & 0.041051 & 0.014808 & 0.005146 \\
1000000 & 0.619961 & 0.265640 & 0.107393 & 0.041162 & 0.014886 & 0.005187 \\
exact & 0.619915 & 0.264757 & 0.106251 & 0.040303 & 0.014525 & 0.004997 \\
\hline
\end{tabular}

TABLE 4

\begin{tabular}{l|llllll}
\hline \hline$u$ & 0 & 2 & 4 & 6 & 8 & 10 \\
\hline mean & 0.619790 & 0.264067 & 0.105692 & 0.040024 & 0.014316 & 0.004799 \\
std. dev. & 0.007323 & 0.009806 & 0.007197 & 0.004605 & 0.002652 & 0.001478 \\
\hline
\end{tabular}

Table 5 and Table 6 show results for the case where the jump amount distribution is gamma with mean equal to 1 and variance equal to 10 (the values of $\lambda, c$ and $\delta$ are the same as in Table 1). This distribution is heavily skewed to the right. It is interesting to observe that for low values of $u$, the probability of ruin is smaller than that of Table 1. It comes as no big surprise that the opposite is true for sufficiently high values of $u$. Note that the convergence of the estimates is quite slow, but it must be kept in mind that this is a "worst case scenario" example since the claims 
distribution is very skewed and no security margin is included in the premium. As can be seen by comparison of Table 2 and Table 4, a higher security margin pulls the storage process faster towards level 0 , thereby inducing a smaller standard deviation for the estimates.

TABLE 5

THE PROBABILITY OF RUIN OBTAINED BY SIMULATION WITH

$$
\lambda=1, c=1, \delta=0.05, P^{\prime}(x)=\frac{(0.1)^{0.1}}{\Gamma(0.1)} x^{-0.9} e^{-0.1 x}, x>0 \text {. }
$$

\begin{tabular}{l|llllll}
\hline$n \backslash u$ & 0 & 2 & 4 & 6 & 8 & 10 \\
\hline 10000 & 0.689627 & 0.534063 & 0.426268 & 0.339340 & 0.269248 & 0.215301 \\
100000 & 0.694396 & 0.543978 & 0.438372 & 0.352647 & 0.283304 & 0.227501 \\
200000 & 0.692600 & 0.542045 & 0.437333 & 0.352160 & 0.283047 & 0.227270 \\
300000 & 0.690774 & 0.539260 & 0.434332 & 0.349699 & 0.281290 & 0.225822 \\
400000 & 0.690506 & 0.539206 & 0.434469 & 0.350262 & 0.282013 & 0.226767 \\
500000 & 0.690252 & 0.538565 & 0.433786 & 0.349681 & 0.281509 & 0.226364 \\
600000 & 0.691321 & 0.539879 & 0.434928 & 0.350625 & 0.281986 & 0.226477 \\
700000 & 0.691120 & 0.539932 & 0.434990 & 0.350448 & 0.281681 & 0.226102 \\
800000 & 0.691508 & 0.540416 & 0.435668 & 0.351265 & 0.282542 & 0.226926 \\
900000 & 0.691812 & 0.540971 & 0.436270 & 0.351890 & 0.283268 & 0.227778 \\
1000000 & 0.692597 & 0.541912 & 0.437225 & 0.352825 & 0.284139 & 0.228484 \\
\hline
\end{tabular}

TABLE 6

\begin{tabular}{l|llllll}
\hline$u$ & 0 & 2 & 4 & 6 & 8 & 10 \\
\hline mean & 0.694120 & 0.544448 & 0.440406 & 0.356273 & 0.287755 & 0.231561 \\
std. dev. & 0.013900 & 0.017105 & 0.017434 & 0.017072 & 0.016591 & 0.015668 \\
\hline
\end{tabular}

\section{Remark}

The special case where $c=0$ in $(5.1)$, i.e. $c(u)=\delta u$, is of unexpected interest. In this case, the process $\{X(t)\}$ will never attain the barrier 0 , and there is an explicit expression for its value at time $t$ :

$$
X(t)=x e^{-\delta t}+\int_{0}^{t} e^{-\delta(t-\tau)} d S(\tau)
$$

The stationary distribution is the distribution of the sum of the discounted claims, that is

$$
F(x)=\operatorname{Pr}\left(\int_{0}^{x} e^{-\delta t} d S(t) \leq x\right) .
$$

For this case, the duality has already been noted by GERBER (1971). Finally, we note that the distribution (5.11) also contains important information about the probability of ruin in the general case where $c>0$ in (5.1). See Example 1.2 in Chapter 3 of Gerber (1979). 


\subsection{Premiums by layers}

Now we look at the case where

$$
c(u)= \begin{cases}c_{0}, & 0=u_{0} \leq u \leq u_{1} \\ c_{1}, & u_{1}<u \leq u_{2} \\ \vdots & \\ c_{k-1}, & u_{k-1}<u<u_{k}=\infty\end{cases}
$$

The premiums vary according to the level of the current surplus. When $c_{0}, c_{1}, \ldots$ is a decreasing sequence, one reasoning that can justify the structure of (5.12) is the following. The greater the surplus of a company, the less the risk of ruin. Thus, a company can take advantage of this situation to lower its premium rates (and thus be more competitive) when its surplus increases. Another reasoning is to view the reduction of premium as a form of dividend payment, where the dividend rate increases along with the surplus. Increasing patterns of $c(u)$ have already been explained in section 5.1 .

Consider the process $\{X(t)\}$ with the premium function given by (5.12). Let $Y_{n}$ and $T_{n}$ be simulated as in section 5.1 and $X_{n}$ be the value of the process immediately after jump $Y_{n}$. A bit of notation must be introduced to show how to apply the method of section 4 .

We will say that a value $x$ is at level $i$ if $u_{i}<x \leq u_{i+1}$. Let

- $b(x)$ be the level of a value $x$, i.e., $b(x)=i$ if $u_{i}<x \leq u_{i+1}$,

- $t_{i}(x)$ be the time needed, starting from $x$, to get to the bottom of level $i \leq b(x)$, i.e.

$$
t_{i}(x)= \begin{cases}\frac{x-u_{b(x)}}{c_{b(x)}} & \text { if } i=b(x), \\ \frac{x-u_{b(x)}}{c_{b(x)}}+\frac{u_{b(x)}-u_{b(x)-1}}{c_{b(x)-1}}+\ldots+\frac{u_{i+1}-u_{i}}{c_{i}} & \text { if } i<b(x),\end{cases}
$$

- $e(x, t)$ be, for a path starting at $x$, the level of the process at time $t$ (assuming no jumps occurred), i.e.

$$
e(x, t)= \begin{cases}0 & \text { if } t>t_{0}(x) \\ \max \left\{i \in\{0,1, \ldots, b(x)\}: t-t_{i}(x) \leq 0\right\} & \text { if } t \leq t_{0}(x)\end{cases}
$$

Thus we have

$$
X_{n}= \begin{cases}\max \left(0, Y_{n}\right) & \text { if } T_{n}>t_{0}\left(X_{n-1}\right) \\ \max \left(0, u_{e}+c_{e}\left(t_{e}-T_{n}\right)+Y_{n}\right) & \text { if } T_{n} \leq t_{0}\left(X_{n-1}\right) .\end{cases}
$$

where $e$ in (5.15) is given by $e\left(X_{n-1}, T_{n}\right)$. Before the $(n+1)$ st jump, the path is below level $x$ for an amount of time

$$
\text { (5.16) } D_{n+1}(x)= \begin{cases}D_{n}(x)+T_{n+1}, & X_{n} \leq x, \\ D_{n}(x)+\left[T_{n+1}-\left(t_{b(x)}\left(X_{n}\right)-\frac{x-u_{b(x)}}{c_{b(x)}}\right)\right]_{+}, & X_{n}>x,\end{cases}
$$

and with (4.2), the probability of ruin can be retrieved. 
An illustration is given in Table 7. For the simulation, the Poisson parameter was equal to 1 and the claims were distributed according to a translated gamma distribution (claims $>-1$ ) with mean equal to 1 and variance equal to 2 . The premiums and layers are as follows:

$$
c(x)= \begin{cases}1.7, & 0 \leq x \leq 2 \\ 1.6, & 2<x \leq 4 \\ 1.5, & 4<x \leq 6 \\ 1.4, & 6<x \leq 8 \\ 1.3, & 8<x \leq 10 \\ 1.2, & x>10\end{cases}
$$

TABLE 7

THE PROBABILITY OF RUIN OBTAINED BY SIMULATION WITH $\lambda=1, P^{\prime}(x)=(x+1) e^{-(x+1)}, x>-1$ AND $c(\cdot)$ GIVEN BY $(5.17)$.

\begin{tabular}{l|llllll}
\hline$n \backslash u$ & 0 & 2 & 4 & 6 & 8 & 10 \\
\hline 10000 & 0.754924 & 0.483182 & 0.293354 & 0.168664 & 0.095282 & 0.051425 \\
100000 & 0.75978 & 0.497156 & 0.303036 & 0.17568 & 0.098522 & 0.054574 \\
200000 & 0.760895 & 0.498213 & 0.303498 & 0.175486 & 0.097763 & 0.053552 \\
300000 & 0.763933 & 0.502501 & 0.306681 & 0.177508 & 0.098657 & 0.053784 \\
400000 & 0.764685 & 0.503882 & 0.307947 & 0.178021 & 0.098809 & 0.053752 \\
500000 & 0.764652 & 0.504126 & 0.308297 & 0.178533 & 0.099493 & 0.054241 \\
600000 & 0.76482 & 0.503911 & 0.307887 & 0.178048 & 0.099054 & 0.053717 \\
700000 & 0.764248 & 0.503313 & 0.307358 & 0.177684 & 0.098852 & 0.05353 \\
800000 & 0.763817 & 0.50263 & 0.306994 & 0.177603 & 0.098887 & 0.053637 \\
900000 & 0.763825 & 0.50262 & 0.307235 & 0.178197 & 0.099494 & 0.054179 \\
1000000 & 0.762866 & 0.501131 & 0.305775 & 0.17693 & 0.098507 & 0.053432 \\
\hline
\end{tabular}

TABLE 8

\begin{tabular}{l|llllll}
\hline$u$ & 0 & 2 & 4 & 6 & 8 & 10 \\
\hline mean & 0.762935 & 0.502425 & 0.308143 & 0.178967 & 0.099972 & 0.054582 \\
std. dev. & 0.009878 & 0.014930 & 0.015546 & 0.013561 & 0.010778 & 0.008359 \\
\hline
\end{tabular}

\section{DISCUSSION OF THE METHOD}

A question has been left unanswered: do other methods exist to obtain the probability of ruin, and if so, are they more efficient (in the sense of speed of convergence)? The answer can be yes or no. Obviously, for cases where an explicit solution is available, the question is not worth asking. When no explicit formula is available, another method is to solve the appropriate integral equation numerically, A few tests show that it is a much faster technique. However, numerical evaluation cannot provide an answer when claim amounts can be negative. In fact, no other method seems to be able to deal with possible negative claim amounts. In that sense simulation is the most general solution. 
The author wishes to point out that, in practice, an actuary does not need an estimate precise to many digits. What he wants is a reliable idea of this value. So in light of the examples given in section 5, simulating 10000 claims can be sufficient. Moreover, he will not need to know the values of $\psi(\cdot)$ for many $u$ 's since he will be needing an answer for a given surplus. With that in mind, the question of efficiency is not a crucial matter for the practitioner because the required time for such simulations is a matter of seconds, even for random variables which are time consuming to simulate (on a Pentium-90 PC with the APL programming language). Since simulation algorithms are nowadays readily available, the method suggested here is easy to implement. However, for extensive studies of $\psi(\cdot)$ when positive claims are sufficient, numerical evaluation is much faster. See PETERSON (1989) for an example of such methods.

\section{ACKNOWLEDGEMENTS}

The author is grateful to Professors François Dufresne and Hans U. Gerber for their helpful comments.

\section{REFERENCES}

Asmussen, S. and Petersen, S.S. (1988) Ruin probabilities expressed in terms of storage processes. Advances in Applied Probability, 913-916.

Dufresne, F. and Gerber, H.U. (1989) Three methods to calculate the probability of ruin. ASTIN Bulletin, 19, 71-90.

FELLER, W. (1971) An introduction to probability theory and its applications. Volume 2, Wiley, New York.

Gerber, H.U. (1971) Der Einfluss von Zins auf die Ruinwahrscheinlichkeit. Bulletin of the Swiss Association of Actuaries, 63-70.

GERBER, H. U. (1975) The surplus process as a fair game-utilitywise. ASTIN Bulletin, 8, 307-322.

GERBER, H. U. (1979) An introduction to mathematical risk theory. S.S. Huebner Foundation, University of Pennsylvania. Philadelphia.

Harrison, J.M. and RESNick, S.I. (1978) The recurrence classification of risk and storage processes. Mathematics of Operations Research, 3, 57-66.

Hoel, P.G., Port, S. C. and Stone, C. I. (1972) Introduction to stochastic processes. Houghton Mifflin, Boston.

Petersen, S.S. (1989) Calculation of ruin probabilities when the premium depends on the current reserve. Scandinavian Actuarial Journal, 147-159.

Ross, S.M. (1983) Stochastic processes. Wiley, New York.

Rudin, W. (1976) Introduction to mathematical analysis. Third edition, McGraw-Hill, New York.

SchmidLI, H. (1994) Risk theory in an economic environment and Markov processes. Bulletin of the Swiss Association of Actuaries, 51-70.

SEAL, H. L. (1972) Risk theory and the single server queue. Bulletin of the Swiss Association of Actuaries, $171-178$.

Segerdahl, C. O. (1942) Über einige risikotheoretische Fragestellungen. Scandinavian Actuarial Journal, 25, 43-83.

SIEGMUND, D. (1976) The equivalence of absorbing and reflecting barrier problems for stochastically monotone Markov processes. The Annals of Applied Probability, 4, 914-924.

TAYLOR, G.C. (1980) Probability of ruin with variable premium rate. Scandinavian Actuarial Journal, $57-76$.

Frederic Michaud

École des $H E C$

Université de Lausanne

CH-1015 Lausanne. Switzerland 